Propagation of rarefaction pulses in particulate materials with strain-softening behavior

E. B. Herbold, V. F. Nesterenko

July 29, 2011

Shock Compression of Condensed Matter - 2011

Chicago, IL, United States

June 26, 2011 through July 1, 2011 
This document was prepared as an account of work sponsored by an agency of the United States government. Neither the United States government nor Lawrence Livermore National Security, LLC, nor any of their employees makes any warranty, expressed or implied, or assumes any legal liability or responsibility for the accuracy, completeness, or usefulness of any information, apparatus, product, or process disclosed, or represents that its use would not infringe privately owned rights. Reference herein to any specific commercial product, process, or service by trade name, trademark, manufacturer, or otherwise does not necessarily constitute or imply its endorsement, recommendation, or favoring by the United States government or Lawrence Livermore National Security, LLC. The views and opinions of authors expressed herein do not necessarily state or reflect those of the United States government or Lawrence Livermore National Security, LLC, and shall not be used for advertising or product endorsement purposes. 


\title{
PROPAGATION OF RAREFACTION PULSES IN PARTICULATE MATERIALS WITH STRAIN-SOFTENING BEHAVIOR
}

\author{
E.B. Herbold ${ }^{1}$, V.F. Nesterenko ${ }^{2,3}$ \\ ${ }^{1}$ Lawrence Livermore National Laboratory, L-236, P.O. Box 808, Livermore, CA, 94550, USA \\ ${ }^{2}$ Department of Mechanical Engineering, UC, San Diego, La Jolla, CA 92093-0411, USA \\ ${ }^{3}$ Materials Science \& Engineering Program, UC, San Diego, La Jolla, CA 92093-0412, USA
}

\begin{abstract}
We investigate rarefaction waves in nonlinear periodic systems with a 'softening' power-law relationship between force and displacement to understand the dynamic behavior of this class of materials. A closed form expression describing the shape of the strongly nonlinear rarefaction wave is exact for $n=1 / 2$ and agrees well with the shape and width of the pulses resulting from discrete simulations. A chain of particles under impact was shown to propagate a rarefaction pulse as the leading pulse in initially compressive impulsive loading in the absence of dissipation. Compression pulses generated by impact quickly disintegrated into a leading rarefaction solitary wave followed by an oscillatory train. Such behavior is favorable for metamaterials design of shock absorption layers as well as tunable information transmission lines for scrambling of acoustic information.
\end{abstract}

Keywords: strongly nonlinear wave, softening behavior, rarefaction wave

PACS: 05.45.Yv, 46.40.Cd, 43.25.+y, 45.70-n

\section{INTRODUCTION}

It is well known that certain materials exhibit an elastic or viscoelastic softening behavior under load. Discrete periodic materials with a "normal" powerlaw relationship between force and displacement, $F \propto \delta^{n}$ for $n>1$ (elastic strain hardening), have been shown to support compression solitary waves in periodic granular assemblies [1-11]. When a dynamic force is significantly larger than the initial force applied to grains, these materials are considered strongly nonlinear and recent investigations have considered their use as information carriers and waveguides $[3,4,8]$. The long wave approximation of the equations of motion for a discrete chain supports a stationary wave solutions traveling in one-dimensional chains or ordered two and three-dimensional arrays of particles in the absence of dissipation [1,2]. It was proven in [2] that stationary solitary and shock waves should form in discrete materials with a general force-displacement relation that stiffens with displacement. Conversely, a discrete chain with an interaction law exhibiting general softening behavior (first considered in [12]) supports rarefaction solitary shock-like waves [2]. In the case of discrete softening materials without tensile strength, the propagation of fracture waves follow directly behind rarefaction pulses.

We investigate the behavior of stationary rarefaction/release waves in discrete periodic materials composed of point masses and an elastically softening interaction law. An exact solution of the long wave approximation with $n=$ $1 / 2$ is presented for stationary rarefaction solitary waves and is compared to numerical simulations. Stationary rarefaction waves have also been investigated in magnetized Hall plasmas and are thought to explain observed anomalous behavior due to a changing electric field [13].

A softening behavior (decreasing of elastic modulus with strain) is observed under certain conditions of loading in a wide range of materials from polymer foams [14] and rubber [15] to actin networks in biological tissues [16]. In general, the response of materials with a "softening" behavior share several common responses under compressive loading: a viscoelastic softening behavior 
characteristic of configuration changes in polymer chains [15] or the collapse of cell-wall structures in polymer foams [14] followed by a stiffening behavior attributed to the bulk resistance to further deformation. Here, we consider a non dissipative interaction law between particles in a onedimensional lattice. In experiments this may be realized for foams where the softening behavior is due to reversible elastic collapse of cell walls. Particle motion may be described by a function of relative particle displacements $\phi\left(u_{\mathrm{i}}-u_{\mathrm{i}+1}\right)$, where $u_{\mathrm{i}}$ is the displacement. The system of discrete equations for a chain of identical particles with a power law potential is

$$
\ddot{u}_{i}=A_{i-1, i} \varphi_{i-1, i}-A_{i, i+1} \varphi_{i, i+1},
$$

where $A$ is an effective stiffness constant and $\varphi_{i-1, i}=\left(u_{i-1}-u_{i}\right)^{n}$ for $0<n<1$. Initial displacements caused by an external force may also be included in displacement $u_{\mathrm{i}}$. The conditions for propagating stationary rarefaction waves in generalized discrete "softening" materials (and with a specific powerlaw interaction) are presented in [2].

The long-wave approximation for Eq. (1) is introduced in a way similar to the case of elastic hardening materials (e.g. particle contact interaction with a Hertzian potential) by assuming that the particle diameter, $a$, is significantly less than the propagating wavelength $L$. The result from applying the long wave approximation to Eq. (1) is,

$u_{t t}=-c_{n}^{2}\left\{\left(-u_{x}\right)^{n}+\frac{n a^{2}}{6(n+1)}\left[\left(-u_{x}\right)^{(n-1) / 2}\left(\left(-u_{x}\right)^{(n+1) / 2}\right)\right]\right\} \underbrace{}_{x}$.

A derivation of Eq. (2) can be found in [2]. The long wave sound speed $c_{0}$ in the chain of particles is found through the linearization of Eq. (2), $c_{0}=c_{n} \sqrt{n} \xi_{0}^{(n-1) / 2}$. Stationary solitary rarefaction waves may propagate in a discrete chain of particles if the chain is initially subject to a constant compression force, $f_{0}$, creating an initial strain $\xi_{0}$. The reduction of Eq. (2) assumes a stationary wave propagating with speed $V$,

$$
y_{\eta \eta}+y-y^{-(n-3) /(n+1)}+y^{-(n-1) /(n+1)} C_{2}=0,
$$

for arbitrary values of $n$. In Eq. (3), $y$ is a reduced form of the strain $\left(\xi=-u_{x}\right), y=\left(c_{n} / V\right)^{(n+1) /(n-1)} \xi^{(n+1) / 2}$, where $c_{\mathrm{n}}{ }^{2}=A a^{\mathrm{n}+1}$ is a parameter with units of speed and $\eta$ is the normalized coordinate traveling with the speed of the solitary wave $V, \eta=x / a \sqrt{6(n+1) / n}$.

Eq. (3) can be rewritten for a nonlinear oscillator moving in an effective "potential field", $d^{2} y / d \eta^{2}=-d W / d y$, where $W(y)$ is defined as

$$
W(y)=\frac{1}{2} y^{2}-\frac{n+1}{4} y^{4 /(n+1)}+C_{3} y^{2 /(n+1)} .
$$

An analogy can be made that a "particle" in the "potential field" $W(y)$ moves from its initial position $\left(y_{1}\right.$, corresponding to $\left.\xi_{0}\right)$ to the position in the wave corresponding to $y_{\min }$ (related to $\xi_{\min }$ ) and back to $y_{1}$. In general, $y_{1}$ corresponding to the case when a minimum strain is equal zero may be expressed only as a function of the power-law exponent $y_{1}=(2 n / n+1)^{(1+n) / 2(1-n)}[2,3]$.

For an anomalous softening interaction between particles, $0<n<1$, rarefaction solitary waves exist when $C_{3}=2 C_{2} /(n+1)$ is bound by $[2,12]$,

$$
\frac{n^{2}-1}{2} n^{n /(1-n)}<C_{3} \leq \frac{n-1}{2}\left(\frac{2 n}{n+1}\right)^{n /(1-n)} \text {. }
$$

The value of $C_{3}$ defines system behavior between weakly and strongly nonlinear regimes by the lower and upper bound of $C_{3}$, respectively. The width of the wave increases in the weakly nonlinear case compared to the strongly nonlinear case.

The exact solution for the long wave approximation can be found for the case where the minimum strain is equal to zero and $C_{3}$ is given by the maximum value in Eq. (5). In the system of reference moving with the wave and centered at the minimum value of strain, an exact solution can be obtained for $n=1 / 2$ and $C_{3}=-1 / 6$ by solving Eq. (3),

$$
y=(2 / 3)^{3 / 2} \mid \tanh ^{3}(\sqrt{2} \eta / 6) \text {. }
$$

The corresponding equation for the strain is,

$$
\xi=\xi_{0} \tanh ^{4}(x / a)
$$

The exact solution Eq. (7) predicts a symmetric 
pulse that starts from the initial strain value $\xi_{0}$, decreases to zero and then returns. The characteristic pulse length is equal to $7 a$ (for a cutoff of $\xi / \xi_{0}=0.98$ ) and does not depend on the amplitude of the solitary wave similar to the case for compressive solitary waves in a "sonic vacuum" where $n>1$ [2].

Fig. 1 shows that the width of the solitary rarefaction wave increases with $0<n<1$. A closed form expression for solitary may be constructed for rarefaction waves for general powers of $n$. The amplitude is equal to $y_{1}$ and the width of the wave increase for values of $n$ in the interval $0<n<1$,

$$
y=\left(\frac{2 n}{n+1}\right)^{(1+n) / 2(1-n)}\left|\tanh ^{(1+n) / n}\left(\frac{\eta}{\sqrt{3(n+1) /(n(1-n))}}\right)\right| .
$$

The two remaining comparisons in Fig. 1 are made between Eq. (8) and the numerical solution of Eq. (3) for $n=1 / 5$ and $4 / 5$. The amplitudes of each pulse from Eq. (8) are the same, but the widths of the pulses are slightly underestimated. However, Eq. (8) is a simple closed form approximation of the general form for the long wave approximation.

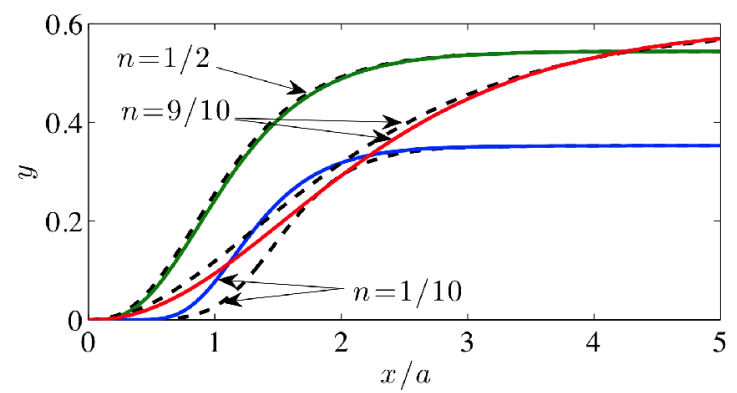

Figure 1. (color online) Equation (6) is compared with the numerical solution of Eq. (3) for three different values of $n$. The amplitudes of the solitary rarefaction waves are exact, but the widths of the pulses increase for $0<n<1$.

It is interesting to find the relationships between the phase speed $V$ and the strain $\xi$. The phase speed can be found using the properties of the potential function; $W\left(y=y_{\min }\right)=W\left(y=y_{1}\right)$ and $\partial W /\left.\partial y\right|_{y=y_{1}}=0$. The speed of the rarefaction wave is [2],

$$
V_{r}=\frac{c_{n}}{\xi_{0}-\xi_{\min }}\left\{\frac{2\left[n \xi_{0}^{n+1}+\xi_{\min }^{n+1}-(n+1) \xi_{0}^{n} \xi_{\min }\right]}{n+1}\right\}^{1 / 2} .
$$

The rarefaction wave with a minimum strain $\left(\xi_{\min }\right)$ equal to zero is a special case where the long wave sound speed represented by $c_{0}$ at the point of zero strain is infinite for $n<1$, but the solitary rarefaction wave speed is finite. We consider the validity of the rarefaction solitary wave solution in long wave approximation with this singularity point by comparison with results for discrete chain. It should be mentioned that a similar situation exists for the case with strongly nonlinear compressive solitary waves in "normal" material $(n>1)$.

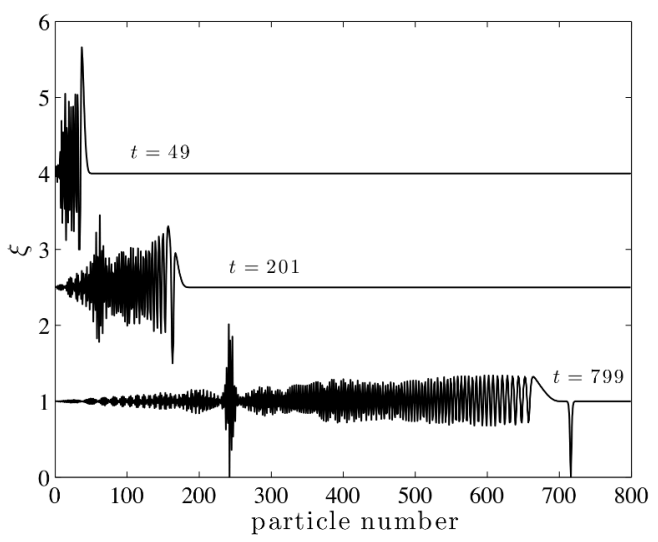

Figure 2. Solitary rarefaction strain wave in a chain of 800 particles compressed with a static force of 1 $\mathrm{N}$ and $n=3 / 4$ impacted by one particle at $6 \mathrm{~m} / \mathrm{s}$. The initial disturbance disintegrates into wave packets, periodic waves and the leading rarefaction solitary wave after travelling $\sim 600$ particles. The strain is offset by 1.5 for each time for visual clarity.

Numerous numerical investigations using $n>1$ agree well with the results obtained using the longwave approximation $[2,5-9]$. For example, the classical Hertzian interaction between perfectly elastic spherical particles is a special case of Eq. (3) where $n=3 / 2$. Additionally, the ratio of the solitary wave speed, $V_{s, r}$ to the sound speed $c_{0}$ is infinite. This ratio is positive $V_{s, r} / c_{0}>1$ for $0<n<1$, meaning the solitary rarefaction wave speed is supersonic [2].

In Fig. 2 impact velocity of $6 \mathrm{~m} / \mathrm{s}$ was specified for the first particle to which a constant force is 
applied. The initial compression pulse quickly diminishes due to nonlinear dispersion and the first rarefaction pulse immediately behind it eventually becomes the leading pulse. It is quite unusual behavior since impact loading is expected to result in compression pulse for "normal" materials. At this impact speed, the resulting minimum strain value of the leading rarefaction pulse is equal to $\xi_{\min }=0$ with $\xi_{0}=1$ with a measured velocity of 0.94 $\mathrm{m} / \mathrm{s}$, compared to $0.93 \mathrm{~m} / \mathrm{s}$ from Eq. (9) with $c_{n}=1$ and $n=3 / 4$, which is within $2 \%$.

The agreement between the speed of the leading pulses of the discrete system of particles and the results from the long wave approximation is excellent for the special case mentioned above. It is interesting that solitary rarefaction waves may arise by specifying a velocity toward or away from the rest of the chain and suggests different methods to test materials experimentally.

The closed form exact and approximate solutions given in Eq. (6)-(8) are in a good agreement with the discrete simulations for different values of $n$.

\section{CONCLUSIONS}

We investigated rarefaction waves in nonlinear periodic systems with a 'softening' forcedisplacement relationship. A closed form expression describing the shape of the strongly nonlinear rarefaction wave is found for $n=1 / 2$. The width of the exact solution does not depend on the amplitude of the strongly nonlinear solitary rarefaction wave and it is smallest for $n=1 / 2$ among investigated values of $n$. The agreement between the supersonic pulse speed of the waves predicted from the theory and numerical calculations is within $2 \%$. A chain of particles under impact was shown to propagate a rarefaction pulse as the leading pulse in initially compressive impulsive loading in the absence of dissipation.

\section{ACKNOWLEDGEMENTS}

The authors wish to acknowledge the support of this work by the U.S. NSF (Grant No. DCMS03013220).

\section{REFERENCES}

1. Nesterenko, V.F., "Propagation of Nonlinear Compression Pulses in Granular Media", Prikl. Mekh. Tekh. Fiz. 5, 136 (1983) [J. Appl. Mech. Tech. Phys., 5, 733 (1984)].

2. Nesterenko, V.F., Dynamics of Heterogeneous Materials (Springer-Verlag, New York, 2001).

3. Nesterenko, V.F., "Nonlinear Waves in "Sonic Vacuum", Fizika Goreniya i Vzryva 28, 121 (1992).

4. Friesecke, G. and Wattis, J.A.D., "Existence Theorem for Solitary Waves on Lattices", Commun. Math. Phys. 161, 391 (1994).

5. Coste, C., Falcon, E., and Fauve, S., "Solitary Waves in a Chain of Beads Under Hertz Contact", Physical Review E 56, 6104 (1997).

6. Manciu, M., Sen, S., and Hurd, A.J., "Impulse propagation in dissipative and disordered chains with power-law repulsive potentials", Physica D 157, 226 (2001).

7. Rosas, A. and Lindenberg, K., "Pulse velocity in a granular chain", Physical Review E 69, 037601 (2004).

8. Daraio, C., Nesterenko, V.F., Herbold, E.B., and Jin, S., "Strongly Nonlinear Waves in a Chain of Teflon Beads", Phys. Rev. E 72, 016603 (2005).

9. Herbold, E.B., and Nesterenko, V.F., "Shock Wave Structure in a Strongly Nonlinear Lattice with Viscous Dissipation", Phys. Rev. E 75, 021304 (2007).

10. Herbold, E.B. and Nesterenko, V.F., "Solitary and shock waves in discrete strongly nonlinear double powerlaw materials", Appl. Phys. Lett. 90, 261902 (2007).

11. Herbold, E.B. and Nesterenko, V.F., "Solitary and Shock Waves in Strongly Nonlinear Metamaterials", in Shock Compression of Condensed Matter, 2008 (M. Elert, M.D. Furnish, R. Chau, N. Holmes, and J. Nguyen, eds.), part I, pp. 231-234.

12. Nesterenko, V.F., Fizika Goreniya i Vzryva,, "Solitary Waves in Discrete Medium with Anomalous Compressibility", 29, 134 (1993).

13. Chuvatin, A.S., Ivanov, A.A., and Rudakov, A.I., "Stationary Rarefaction Wave in Magnetized Hall Plasmas", Phys. Rev. Lett. 92, 095007 (2004).

14. Klintworth, J.W., and Stronge, W.J., "Elasto-plastic yield limits and deformation laws for transversely crushed honeycombs", Int. J. Mech. Sci. 30, 273 (1988).

15. Ogden, R.W., "Large deformation isotropic elasticityon the correlation of theory and experiment for incompressible rubberlike solids", Proc. R. Soc. Lond. A. 326, 565 (1972).

16. Chaudhuri, O., Parekh, S.H., and Fletcher, D.A., "Reversible stress softening of actin networks", Nature 445, 295 (2007).

This work performed under the auspices of the U.S. Department of Energy by Lawrence Livermore National Laboratory under Contract DE-AC52-07NA27344. 